\title{
Transtorno de Déficit de Atenção e Hiperatividade: Um Olhar no Ensino Fundamental
}

\section{Attention Deficit Hyperactivity Disorder: A Glance at the Elementary School}

\author{
Graciela Inchausti de Jou*, ${ }^{*}$, Bruna Amaral ${ }^{b}$, Carolina Robl Pavan $^{b}$, \\ Luiziana Souto Schaefer ${ }^{b} \&$ Marilene Zimmer ${ }^{c}$ \\ ${ }^{a}$ Universidade Federal do Rio Grande do Sul \\ ${ }^{b}$ Pontifícia Universidade Católica do Rio Grande do Sul \\ ${ }^{c}$ Universidade Federal do Rio Grande
}

\begin{abstract}
Resumo
Nos últimos anos, o diagnóstico de TDAH aumentou no contexto escolar, aumentando também a quantidade de pesquisas sobre o assunto. O presente estudo investigou o número de alunos com TDAH em 17 escolas de Porto Alegre e a percepção de 136 professores de $1^{\mathrm{a}}$ à $8^{\mathrm{a}}$ série, sobre o transtorno e o comportamento das crianças com TDAH. Dois questionários foram utilizados: um preenchido pela direção da escola e o outro, pelos professores. A média dos alunos com TDAH das escolas estudadas foi de 3\%, com dois casos extremos: uma escola com $51 \%$ e outra com $0,2 \%$. Em relação à percepção dos professores, houve discrepância entre os possíveis casos apontados por estes e os levantados junto à direção. Concluise, portanto, que é necessário oferecer ao ambiente escolar mais informação sobre esse transtorno, pois a maioria das escolas estudadas não oferece subsídios aos professores.

Palavras-chave: Transtorno de déficit de atenção; Ensino fundamental; Aprendizagem.
\end{abstract}

\begin{abstract}
In the last years the Attention Deficit Hyperactive Disorder (ADHD) has increased in schools. This study investigates the number of students reported with the ADHD in 17 schools in Porto Alegre, a city in the south of Brazil. One hundred and thirty six $1^{\text {st }}$ to $8^{\text {th }}$ grade teachers were asked about their perceptions concerning this behavioral disorder. Two questionnaires filled in by the school direction and by the teachers, respectively, were used. The average of students with ADHD in the schools was 3\% - one school reported $51 \%$ and the other $0.2 \%$. Concerning teachers' perceptions it was found a discrepancy between cases identified as ADHD by the teachers and the cases reported by the school direction. It is necessary to offer more information to the schools on the ADHD since most of them do not provide subsidies for the teachers regarding this matter.

Keywords: Attention deficit hyperactivity disorder; Elementary school; Learning.
\end{abstract}

Devido ao aumento significativo da presença do Transtorno de Déficit de Atenção e Hiperatividade (TDAH) nas escolas, ele tem sido o foco das atenções de pais e educadores. Psicólogos, psiquiatras e neurologistas especializam-se cada vez mais no tratamento e prevenção desse transtorno. Indicam-se remédios e psicoterapias, orientam-se pais e professores, acompanha-se o desenvolvimento da criança e tratam-se as comorbidades. Os casos parecem aumentar dia-a-dia, tomando conta de nossa cultura.

O TDAH é um transtorno do desenvolvimento do autocontrole que afeta a atenção, o controle de impulsos e o nível de atividade (Barkley, 2002). Apesar de existir crianças com TDAH identificadas como apenas desatentas, ou apenas hiperativo-impulsivas, a maioria (62\%)

* Endereço para correspondência: Avenida Ramiro Barcelos, 2600, Bairro Santa Cecília, Porto Alegre, RS, Brasil, CEP90035-003. Email: grajou@terra.com.br corresponde ao tipo combinado (Rohde, 2002). Portanto, a dificuldade de sustentar a atenção é um dos sintomas mais prevalentes nas crianças com TDAH. Socialmente essas crianças, muitas vezes, são percebidas como indisciplinadas, pela própria dificuldade de atentar e de seguir regras.

Como é percebida uma criança com TDAH? Elas são descritas pelos seus pais, professores e colegas como crianças que sonham acordadas, que não escutam, que estão sempre perdendo as coisas, que são esquecidas e facilmente distraídas pelo ambiente, que precisam de constante atenção e que não acabam nada do que começam. Também pela sua dificuldade de controlar seus impulsos, elas são apontadas como impacientes, pois sempre interrompem os outros, respondem antes da hora, não esperam sua vez e tentam sempre fazer atalhos nas suas tarefas. Quanto a sua excessiva motricidade ou hiperatividade, pais e professores comentam que elas têm a tendência de abraçar o mundo, ficam retorcendo-se sem 
conseguir ficar sentadas, falam demais, freqüentemente ficam cantarolando ou fazendo barulhos estranhos, e são incapazes de frear as atividades motoras. Elas, na realidade, não possuem meio termo: ou estão dormindo ou estão pulando.

\section{Prevalência do TDAH}

Com relação à presença do TDAH, várias pesquisas de diferentes países mostram uma prevalência do transtorno entre $3 \%$ e $9 \%$. A diferença entre as médias levantadas nesses estudos seria consequiência do critério utilizado para definir o transtorno (Spencer, Biederman, Wilens, \& Faraone, 2002). Nos EUA, entre a população escolar, o índice é de 3\% a 15\%. Na Alemanha, cerca de 9\% (Facion, 2004); já no Brasil, Rohde et al. (1999) apontam para um índice de 5,8\%. O estudo feito na cidade de Salvador, na Bahia, por Freire e Pondé (2005), informa que as crianças com TDAH em idade escolar chegam a um índice entre 5,5\% e 8,5\%. Os autores acrescentam que a média do transtorno em estudos feitos em comunidade é de $10,3 \%$, tendo os meninos uma prevalência de $9,2 \%$ e as meninas de $3 \%$. Os resultados não mostraram diferença por área geográfica. Entretanto Freire e Pondé (2005) advertem que há certa controvérsia entre as pesquisas com relação às variáveis socioeconômicas.

Polanczyk, Lima, Horta, Biederman e Rohde (2007), em um estudo recente de meta-análise, investigaram a prevalência do TDAH nos cinco continentes, procurando pelas possíveis causas da variabilidade das estimativas do transtorno. Dos 9.105 registros achados nos bancos de dados MEDLINE e PsychINFO os autores revisaram 303 artigos completos e incluíram 102 trabalhos com um total de 171.756 sujeitos. Os dados mostraram uma prevalência de $5.29 \%$ em nível mundial associada a uma variabilidade significativa, mais explicada pelas diferenças metodológicas dos estudos do que pelas diferenças geográficas.

\section{Diagnóstico e Tratamento do TDAH}

O diagnóstico do TDAH fundamenta-se no quadro clínico comportamental, já que não existe um marcador biológico específico que contemple todos os casos desse transtorno. Não existe, portanto, nenhum teste psicométrico, neurológico ou laboratorial que permita diagnosticar o TDAH. O diagnóstico é o resultado da análise de informações obtidas de várias fontes e em diversas situações, incluindo desde a queixa feita no consultório do profissional até as informações obtidas mediante entrevistas e escalas com os pais ou responsáveis, os professores e a anamnese da criança (Guardiola, 2006; Low, 2006; Rotta, 2006).

Barkley (2002) aponta para a importância de realizar um diagnóstico acertado e precoce, pois é através dele que se dá o adequado manejo e tratamento da criança. Deve-se considerar que as crianças do tipo desatento possuem diferentes manifestações das hiperativas, ou das combinadas; exigem, portanto, tratamentos que contemplem essas diferenças. Devido à estreita relação entre o TDAH e comorbidades (Riesgo, 2006) e entre o TDAH e comprometimentos social, emocional, acadêmico e lingüístico (Silva \& Paula Souza, 2005) o diagnóstico precoce permite um melhor prognóstico dos casos, já que, tais aspectos poderiam ser trabalhados desde cedo.

$\mathrm{O}$ tratamento de crianças com TDAH visa à reorganização dos seus comportamentos, viabilizando atitudes funcionais no meio familiar, escolar e social. Com tal finalidade, o tratamento enfatiza a modificação do comportamento, ajustamento acadêmico, atendimento psicoterápico e terapia farmacológica (Rotta, 2006). Dentro da terapia farmacológica, a medicação mais utilizada é o metilfenidato. Segundo vários autores, o uso de medicação se faz necessário ao tratamento do TDAH, uma vez que os estimulantes, as drogas mais comumente utilizadas, têm-se mostrado bastante eficazes na melhora do comportamento, desempenho escolar e ajustamento social para aproximadamente $50 \%$ a $95 \%$ das crianças com TDAH (Barkley, 2002; Rotta, 2006). Contudo, o uso de psico estimulantes para o tratamento do transtorno mantém-se ainda controverso (Landskron, 2007; Ulloa, 2007; Vetter et al., 2008).

Quanto às psicoterapias, a cognitiva comportamental tem mostrado bons resultados ao trabalhar auto-instrução, automonitoramento, auto-avaliação, auto-reforço e instruções comportamentais (Ervin, Bankert, \& DuPaul, 1996).

\section{O TDAH na Escola}

Muitos sintomas do TDAH são observáveis desde muito cedo na infância; entretanto, eles são mais percebidos no início da escola. As dificuldades de atenção e de hiperatividade dessas crianças são reconhecidas pelos professores quando comparadas com as outras crianças da mesma idade. É no contexto escolar que a inquietude e a impulsividade são interpretadas como falta de disciplina e a desatenção como negligencia, apesar de tais comportamentos serem mais relacionados a uma disfunção no desenvolvimento neurológico.

Os professores são freqüentemente a primeira fonte de informação para determinar o diagnóstico do TDAH e, na maioria das vezes, são eles que solicitam uma avaliação profissional para seu aluno. Conseqüentemente, os trabalhos que investigam as experiências, as percepções e o conhecimento dos professores sobre o TDHA (Dias, 2005; Freire \& Pondé, 2005; Glass \& Wegar, 2000; Havey, 2007; Havey, Olson, McCormick, \& Cates, 2005; Landskron, 2007; Marcon, 2006) são de extrema importância para mostrar o que pode ser feito na área educacional. A maioria desses autores concluiu que é escasso o conhecimento dos professores sobre o transtorno, e é fraco o suporte que as escolas oferecem nessa área. 
Jou, G. I., Amaral, B., Pavan, C. R., Schaefer, L. S. \& Zimmer, M. (2010). Transtorno de Déficit de Atenção e Hiperatividade: Um Olhar no Ensino Fundamental.

O estudo de Havey et al. (2005) examinou a percepção de professores sobre causa, incidência e métodos apropriados de tratamento do TDAH. Os resultados mostraram que os educadores identificaram mais casos de transtorno $(23,97 \%)$ do que o esperado, segundo os índices de prevalência no DSM-IV (3\% a 5\%). Com relação à etiologia, os aspectos ambientais estariam influenciando mais a percepção dos professores do que os aspectos neurológicos, mostrando um desconhecimento desta abordagem sobre o transtorno. Em outro estudo, Havey (2007) comparou a percepção de professores holandeses e americanos sobre a causa e a incidência do TDAH. Os dados mostraram que os primeiros pensavam que a etiologia do transtorno estava mais ligada a aspectos bioquímicos, enquanto os professores americanos pensavam que estava mais ligada à combinação de fatores ambientais e bioquímicos. No mesmo estudo, Havey (2007) solicitou aos mestres que estimassem o número de alunos com TDAH em sua aula. Todos os grupos de professores pensavam que havia em suas turmas mais alunos do que aqueles que foram diagnosticados com o transtorno.

Um estudo de prevalência de TDAH, realizado em uma única escola pública primária na cidade de Niterói (Vasconcelos et al., 2003), indicou $17,1 \%$ de crianças com TDAH. A triagem inicial realizada pelas professoras foi positiva para 108 de 403 crianças (26\%). Desses alunos, $69(63 \%)$ tiveram diagnóstico confirmado, mostrando, porém, discrepância entre o parecer das professoras e o diagnóstico confirmado.

Pesquisas sobre a percepção dos professores a respeito do TDAH alertam sobre o quanto eles estão comprometidos com o diagnóstico, o manejo e o tratamento dessas crianças. No processo da educação formal, tanto o educador quanto o educando têm um papel ativo, incrementado pela interação social que passa pelas percepções de ambos. A prática educativa viabiliza, através da interação professor-aluno, transcender o espaço da sala de aula, constituindo-se, também, numa prática social (Aquino, 1996; Frison \& Schwartz, 2002). É do professor que as crianças recebem os comentários de como eles são como alunos, cultivando sua auto-imagem e sua auto-estima. Se as crianças com o transtorno são constantemente apontadas como diferentes, indisciplinadas e desatentas, possivelmente desenvolverão uma baixa auto-estima e uma imagem negativa de si mesmas.

Com relação ao rendimento acadêmico, o baixo desempenho escolar está presente em algumas crianças com TDAH. Considera-se que $20 \%$ das crianças com o transtorno tenham dificuldade de aprendizagem (Poeta $\&$ Rosa Neto, 2004). Nesses casos, se a intervenção demora, esses alunos podem não construir a fundação acadêmica sólida de que precisam para ter sucesso nas séries posteriores. As crianças, além de estarem prontas para aprender, devem ter também oportunidades apropriadas de aprendizagem. Se o sistema educacional não lhes oferece isso, talvez nunca possam desenvolver suas plenas capacidades. Segundo Rohde (1999) e Rohde, Dorneles e Costa (2006), é fundamental que a escola esteja familiarizada com os conceitos básicos do TDAH.

Para crianças com TDAH, a rigidez da sala de aula pode ser fatal. A fim de progredirem, devem ser encorajadas a trabalhar ao seu próprio modo. Assim, as escolas, voltadas de modo exclusivo para resultados em termos de conteúdo, podem não ser um ambiente adequado para essas crianças e adolescentes. Se tais indivíduos forem confiados a um professor inflexível, no que concerne a tarefas e testes, ou que usa materiais e métodos inapropriados às suas necessidades, eles serão reprovados (Smith \& Strick, 2001). A escola, tanto pode tornarse uma instituição estimuladora como, pelo contrário, pode ser fonte de conflitos (Bassedas et al., 1996).

O presente estudo teve como objetivo investigar o TDAH no ambiente escolar. Para tal, abordou quatro aspectos: (a) quantidade de casos e sua distribuição por gênero; (b) as medidas terapêuticas mais utilizadas (uso de medicação, acompanhamento psicoterapêutico, ambos ou nenhum); (c) a percepção dos professores acerca dos comportamentos dos alunos; (d) presença de suporte profissional e capacitação para os professores nas escolas estudadas.

\section{Método}

\section{Participantes}

Participaram 17 escolas de Porto Alegre e sua periferia, sendo 7 públicas e 10 privadas e um total de 136 professores, de $1^{\mathrm{a}}$ a $8^{\mathrm{a}}$ série. Inicialmente, foram contatadas 25 escolas das quais 3 (particulares) não mostraram interesse em participar, 2 (públicas) não devolveram o material e 3 ( 1 privada e 2 públicas) foram descartadas por preenchimento incompleto dos questionários.

\section{Instrumentos}

Um questionário a ser respondido por um responsável da escola para levantar dados específicos de cada escola sobre as crianças com TDAH. Neste questionário, perguntava-se sobre os registros de crianças com TDAH, quantos meninos, quantas meninas, idade, série e tratamento e que medida era tomada quando se tem suspeita de uma criança com esse Transtorno.

Um questionário a ser respondido por professores da $1^{\mathrm{a}}$ à $8^{\mathrm{a}}$ série do ensino fundamental, perguntando sobre sua percepção do TDAH, como eles descreveriam o transtorno e o comportamento desses alunos, em que séries lecionavam, quantos alunos/as com TDAH tinham na aula e qual o suporte da escola nessa área.

Termo de consentimento livre e esclarecido (TCLE).

\section{Procedimentos na Coleta de Dados}

No início, foi agendado telefonicamente um encontro com a diretora ou um responsável de cada uma das 25 escolas públicas e privadas de Porto Alegre e periferia. As escolas formavam parte do banco de dados do grupo 
de pesquisa. Nesse primeiro encontro, explicava-se a pesquisa e convidava-se a escola a participar. Às escolas que mostraram interesse, 17 no total, entregou-se uma pasta com o projeto, o termo de consentimento livre e esclarecido (TCLE) e o questionário a ser respondido. $\mathrm{O}$ responsável assinava o TCLE pela escola e recebia outra pasta com 20 questionários e 20 TCLEs para serem distribuídos, segundo critério de interesse e disponibilidade, entre professores de $1^{\mathrm{a}}$ a $8^{\mathrm{a}}$ série. Combinava-se a data para buscar os questionários preenchidos. Uma vez terminada a pesquisa, ofereceu-se às escolas a possibilidade de devolução dos resultados, junto com uma palestra sobre TDAH. Esta atividade foi oferecida em cada uma das escolas num seminário para professores.

\section{Considerações Éticas}

A presente pesquisa não apresenta riscos de nenhuma índole aos participantes, pois se trata de uma tarefa de investigação, que visa a verificar o número de crianças com déficit de atenção e hiperatividade bem como a percepção dos professores com relação ao transtorno. $\mathrm{O}$ TCLE informa e esclarece aos participantes as atividades que serão realizadas. Especifica também que a identidade será mantida no mais rigoroso sigilo e que, em qualquer momento, a pessoa tem a liberdade de deixar de participar, assim como de perguntar sobre a metodologia utilizada.

\section{Resultados e Discussão}

Os dados foram analisados quantitativamente, em termos de percentagens, e qualitativamente mediante análise de conteúdo (Bardin, 2000). Os resultados e a discussão são apresentados da seguinte maneira: primeiro abordase o número de casos e sua distribuição por gênero. Logo após, se discute a respeito da percepção dos professores sobre o TDAH e o suporte que as escolas oferecem para a capacitação de seus profissionais nesta área. Por último, aborda-se o tipo de tratamento.

O número de alunos do ensino fundamental, nas 17 escolas pesquisadas, foi de 8681 , dos quais $281(3,24 \%)$ com idade média de 10 anos tinham registrado o diagnóstico de TDAH nas suas fichas. Duzentos e vinte sete alunos $(80,8 \%)$ eram meninos e $54(19,2 \%)$ eram meninas. Esta prevalência e sua distribuição por gênero estão de acordo com os dados apontados por Spencer et al. (2002), no entanto, diferem aos apontados por Rohde et al. (1999) que indicaram 5,8\% de prevalência e Freire e Pondé (2005) que indicaram entre 5,5\% e 8,5\%. É importante salientar que a diferença entre as médias levantadas pelos diferentes estudos, geralmente depende das ferramentas metodológicas, ou do próprio critério utilizado para definir o transtorno (Spencer et al., 2002).

Com relação aos resultados do presente estudo, devese considerar que, apesar da média de prevalência ir ao encontro dos dados levantados na literatura, achou-se uma discrepância importante entre algumas escolas. A média de prevalência de todas as escolas foi de $3,2 \%$ e a média de prevalência sem os casos extremos foi de $2,8 \%$. Por exemplo, uma escola registrou $51 \%$ de alunos com TDAH e outra apresentou $0,2 \%$. Tal discrepância foi esclarecida pela coordenadora da última escola, ao apontar que era norma da escola tentar resolver os problemas relacionados às dificuldades de aprendizagem e/ou de disciplina, primeiro com a família e a escola e só depois encaminhar para uma avaliação psiquiátrica. Corroborando essa posição, a orientadora educacional dessa escola frente à pergunta "Que medida é tomada quando se tem suspeita de uma criança com esse Transtorno?" respondeu: "Coleta-se os dados entre os professores e a família. Orientação aos professores de como conduzi-los na rotina escolar. Entrevista com os pais (orientação e encaminhamento). Acompanhamento mais individualizado". Já na escola com registro de $51 \%$ de alunos com TDAH, a orientadora educacional respondeu:

Quando a professora levanta essa suspeita, observase a criança mais de perto, conversa-se com os pais ou responsáveis e encaminha-se para o neuropediatra ou psiquiatra para uma avaliação. Se o diagnóstico for positivo, geralmente é encaminhada para acompanhamento psicológico ou psicopedagógico, conforme o caso.

Os dois casos citados exemplificam claramente as diferentes condutas que as escolas têm logo que surge a suspeita do transtorno nos alunos. No primeiro caso resolve-se, inicialmente, com medidas educacionais, e só quando estas não funcionam, encaminha-se o aluno para uma avaliação profissional. No segundo, porém, quando a professora levanta a suspeita, já se encaminha a criança para uma avaliação profissional. Este fato é considerado como um dos dados mais relevantes do presente estudo, pois leva a refletir sobre quanto o posicionamento das escolas frente ao transtorno influencia na quantidade de casos diagnosticados.

Outro aspecto que pode ser considerado com relação ao índice de prevalência deste estudo é a possibilidade de algumas famílias não ter informado à escola sobre o diagnóstico dos filhos, já que como aponta Landskron (2007) alguns pais poderiam mostrar-se resistentes a aceitar o diagnóstico.

Da mesma forma, vale questionar sobre o quanto os professores estão informados em relação ao transtorno e quanto as escolas investem na capacitação de seu quadro docente, já que, é a percepção do professor que, geralmente, está por trás do encaminhamento da criança para buscar uma solução psicológica adequada.

O presente trabalho também mostrou que os professores apontaram 384 alunos como tendo o transtorno, revelando a discrepância com os 281 casos levantados nas fichas dos alunos. Ao analisar esses dados por escola, observou-se que a discrepância se deu nos dois sentidos, como mostra a Tabela 1, a seguir. Os casos extremos foram constatados na escola 9 , onde os professores apontaram 93 alunos com alto índice de probabilidade 
Jou, G. I., Amaral, B., Pavan, C. R., Schaefer, L. S. \& Zimmer, M. (2010). Transtorno de Déficit de Atenção e Hiperatividade: Um Olhar no Ensino Fundamental.

de ter TDAH e apenas 18 alunos foram reportados pela escola, segundo as fichas. Já na escola 12, os professores indicaram 31 alunos com possibilidade de terem TDAH, mas a escola tinha registro de 53 alunos.

Tabela 1

Discrepância entre a Quantidade de Alunos Informados pela Escola e os Percebidos pelos Professores por Escola

\begin{tabular}{lll}
\hline & Dados da Escola & Dados do Prof. \\
\hline Escola 1* & 2 alunos & 5 alunos \\
Escola 2* & 13 alunos & 10 alunos \\
Escola 3* & 17 alunos & 25 alunos \\
Escola 4* & Não tem dados & Não tem dados \\
Escola 5 & 18 alunos & 17 alunos \\
Escola 6 & 22 alunos & 60 alunos \\
Escola 7 & 27 alunos & 9 alunos \\
Escola 8 & 18 alunos & 6 alunos \\
Escola 9 & 18 alunos & 93 alunos \\
Escola 10* & 6 alunos & 12 alunos \\
Escola 11 & 46 alunos & 30 alunos \\
Escola 12 & 53 alunos & 31 alunos \\
Escola 13 & 12 alunos & 20 alunos \\
Escola 14 & 23 alunos & 29 alunos \\
Escola 15* & 5 alunos & 26 alunos \\
Escola 16* & 7 alunos & 9 alunos \\
Escola 17 & 4 alunos & 2 alunos \\
\hline
\end{tabular}

Nota. $*=$ Escolas Públicas.

O estudo de Freire e Ponde (2005), realizado na cidade de Salvador, com 763 alunos do ensino fundamental, também mostrou essa discrepância. O objetivo da pesquisa consistiu em estimar a prevalência de crianças com TDAH. Através dum inquérito com os professores, mostrou que, para os casos diagnosticados como alta-probabilidade de déficit de atenção, houve um índice de discordância de $47 \%$, entre a percepção inicial do professor e o resultado final da análise das escalas do TDAH. Já para os casos de alta probabilidade de hiperatividade e impulsividade e de problemas de aprendizagem houve $100 \%$ de concordância. Esses resultados apontam para o caráter encoberto que pode ter o déficit de atenção e para o caráter aberto dos comportamentos hiperativos e impulsivos. Isto significa que, muitas vezes, uma criança mais inquieta ou indisciplinada pode adquirir comportamentos equivalentes às crianças com TDAH. Vale lembrar que dados de prevalência apontam para apenas $11,2 \%$ para o subtipo de hiperatividade.

Neste estudo, a maioria dos professores destacou como características das crianças com o transtorno a dificuldade para se concentrar (80\%), não terminar as tarefas (70\%) e ser desatentas $(60 \%)$. Também os professores qualificaram essas crianças como, agitadas, impulsivas, agressivas e com dificuldade de cumprir regras. Três professores mencionaram a desorganização como característica. Uma professora destacou a liderança, enquanto outra observou a apatia e a sonolência. Como conseqüência dessas características todas, foram citadas a baixa auto-estima dos alunos e sua exclusão pelos colegas.

Segundo Rohde et al. (2006), os estudantes com TDAH tipo desatento mostram mais dificuldade nas atividades escolares, já que a atenção seria um fator importante para a qualidade da aprendizagem. Este fato não passou despercebido pelos professores do presente estudo que $80 \%$ deles apontaram a falta de concentração como a principal característica no TDAH.

Com relação ao conceito que os professores têm sobre o TDAH, $85 \%$ o definem por meio de características específicas: não prestam atenção, são inquietos, agitados, briguentos, etc. e $15 \%$ utilizam uma definição formal, como por exemplo:

É um distúrbio comportamental que afeta ao indivíduo, acarretando transtorno, tanto na sociabilidade quanto na aprendizagem, sendo mais perceptível na idade escolar, onde com frequiência, a criança é tratada de desatenta, desligada, desorganizada, problemática . . . até ser finalmente diagnosticada.

A seguir expõem-se outros exemplos de como os professores conceituam o TDAH ao responder à pergunta "Como você descreve o transtorno de déficit de atenção hiperatividade?":

Prof. A: "Falta de atenção, lento ao copiar, sem motivação, nervoso, não consegue ficar parado nem atento".

Prof. B: "É um transtorno neurológico, de causas genéticas que acompanham o indivíduo por toda vida. Os sintomas básicos são: desatenção, inquietude e muitas vezes impulsivos"

Prof. C: "Na faculdade, nunca fomos orientadas para este tipo de problema. Aliás nem aprendemos sobre isso. Acredito que deve ser aquele aluno que não consegue envolver-se com o processo de aprendizagem. Não senta, não corresponde ao esperado pelo professor."'

Acresce dizer que foram poucos os professores (15\%) que mostraram um conhecimento formal sobre o transtorno, confirmando a desconfiança de que a maioria dos professores não está preparada para enfrentar o TDAH. $\mathrm{Na}$ mesma linha, os dados mostraram que apenas 4 escolas das 17 ofereciam cursos, palestras e informações aos professores e trabalhos com os pais. Três não ofereciam nenhum tipo de suporte. Seis escolas (5 privadas e 1 pública) ofereciam reforço pedagógico a seus alunos em geral.

Quanto às medidas terapêuticas mais utilizadas, os dados fornecidos pelas unidades mostraram que $33 \%$ das crianças com TDAH recebiam medicação e acompanhamento psicoterapêutico; $30 \%$ tomavam somente medicação; $26 \%$ tinham apenas acompanhamento psicoterapêutico e $11 \%$ não recebiam nem um nem outro. Ver Figura 1. 


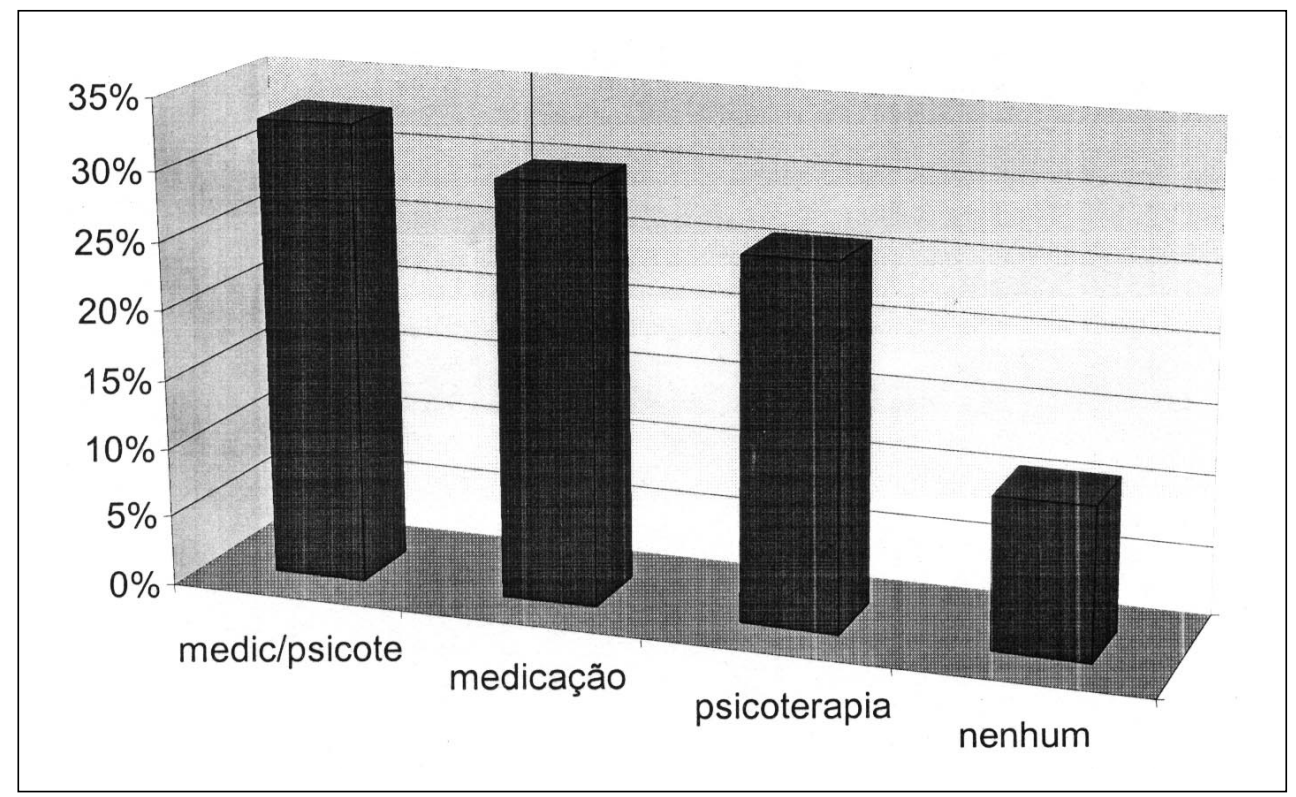

Figura 1. Percentagens das crianças segundo o tratamento

O tratamento mais indicado para o transtorno de TDAH consiste numa abordagem múltipla de intervenções psicossociais e psicofarmacológicas, segundo apontam Rohde, Barbosa, Tramontina e Polanczyk (2000) e Rotta (2006). Este estudo, no entanto, mostrou que apenas $33 \%$ das crianças com o transtorno se beneficiavam da psicoterapia e da terapia farmacológica ao mesmo tempo.

Embora o percentual dos alunos que não recebiam nenhum tipo de ajuda tenha sido bastante baixo (11\%), ainda assim é preocupante saber que essas crianças tenham seu desenvolvimento comprometido por falta de acompanhamento profissional. Diante dessa situação, os pesquisadores foram informados que muitos pais não querem envolver-se com qualquer tipo de tratamento. Da mesma forma, o estudo de Landskron (2007), que analisou as narrativas das professoras sobre o TDAH, mostrou que as mesmas apontaram para a resistência dos pais em aceitar o diagnóstico e, portanto, de procurar um tratamento.

Rohde et al. (2000) sugerem um acompanhamento psicopedagógico, para as crianças com o transtorno, centrado nas dificuldades de aprendizagem, a fim de que possam vir a desenvolver habilidades e competências no planejamento e na organização das suas atividades. Sabe-se que intervenções psicopedagógicas melhoram a qualidade de aprendizagem e, conseqüentemente, o desempenho escolar, e melhora, por sua vez, a auto-estima da criança com TDAH.

Por outro lado, o uso de psicofármacos e TDAH tem propiciado inúmeras pesquisas nos últimos anos (Rohde et al., 2000; Rotta, 2006; Segenreich \& Mattos, 2004), mostrando seus benefícios. Contudo, muito se questiona sobre o aumento do consumo desses medicamentos em todo o mundo, sendo de $600 \%$ desde 1990 (Landskron, 2007).
Os aspectos questionáveis sobre o uso de psicofármacos centram-se nos possíveis efeitos colaterais, no uso adequado ou não para tratar as possíveis comorbidades, na possível discriminação que sofrem os usuários da medicação com dosagens, no período escolar, nas doses adequadas e nos períodos de descanso indicados. Além do mais, surge o perigo do uso alternativo que muitos usuários fazem do metilfenidato.

Com relação aos efeitos colaterais, Vetter et al. (2008) apontam que entre os anos de 1999 e 2003, 25 pessoas, entre elas 19 crianças, tomando medicação para TDAH tiveram morte súbita e 43 pessoas, entre elas 26 crianças, tiveram manifestações cardiovasculares, como derrame, arritmias e palpitações cardíacas. Ainda no mesmo artigo, os autores informam que o TDAH pode ser mais prevalente em crianças com doenças cardiovasculares que na população pediátrica. Estas informações servem de alerta para o diagnóstico e tratamento da síndrome, colocando em evidencia a necessidade de maior rigor nas avaliações e maior exigência de exames cardiológicos antes de iniciar qualquer tratamento.

Mesmo que o TDAH esteja entre os distúrbios psicológicos infantis mais bem estudados, ainda há muito que não se sabe. O TDAH permanece mal compreendido e controverso nas mentes do público em geral e das autoridades educacionais. É importante investigar as possíveis causas do TDAH, mas é importante também investigar as medidas educacionais preventivas e protetoras de comorbidades. Mais importante ainda é capacitar o quadro docente para melhor compreender e diferenciar as características dos alunos, evitando enquadrá-los em falsos rótulos.

Com relação à melhor didática e ao melhor tratamento destas crianças em sala de aula, Rohde et al. (2006) oferecem, entre outras, as seguintes sugestões: explicar 
Jou, G. I., Amaral, B., Pavan, C. R., Schaefer, L. S. \& Zimmer, M. (2010). Transtorno de Déficit de Atenção e Hiperatividade: Um Olhar no Ensino Fundamental.

claramente a finalidade de cada tarefa, manter uma rotina diária com descansos definidos, reforçar visual e auditivamente as regras estabelecidas, dividir as atividades em unidades menores, ensinar a captar a idéia principal, ensinar a monitorar suas atividades.

Analisando detalhadamente essas e outras sugestões encontradas na literatura pertinente, pode-se concluir que elas são medidas vantajosas para todo e qualquer estudante que esteja inserido na sociedade de informação da qual faz parte nossa realidade atual. Possivelmente, a preocupação com a aprendizagem de crianças com TDAH num futuro próximo, provocaria uma mudança de paradigma educacional, invocada por tantos teóricos. Desse modo, as crianças serão ensinadas a gerenciar sua aprendizagem e a lidar com a transitoriedade do conhecimento.

Finalmente, num contexto de educação mais abrangente, cabe a seguinte pergunta: são as crianças que têm dificuldade de aprendizagem ou é o sistema educacional que tem dificuldade de ensinar, numa sociedade que enfrenta grandes mudanças, onde se hiper estimula nossas crianças?

\section{Referências}

Aquino, J. R. G. (1996). A desordem na relação professor-aluno: Indisciplina, moralidade e conhecimento. In J. G. Aquino, Indisciplina na escola: Alternativas teóricas e práticas (pp. 39-55). São Paulo, SP: Summus.

Bardin, L. (2000). Análise de conteúdo. Lisboa, Portugal: Edições 70 .

Barkley, R. A. (2002). Transtorno de déficit de atenção/ hiperatividade - TDA-H. Porto Alegre, RS: Artes Médicas.

Bassedas, E., Huguet, T., Marrodán, M., Oliván, M., Planas, M., Rossell, M., et al. (1996). Intervenção educativa e diagnóstico psicopedagógico (3. ed.). Porto Alegre, RS: Artes Médicas.

Dias, L. M. R. (2005). Programa de estratégias para professores de crianças desatentas, hiperativas/impulsivas. Dissertação de Mestrado não-publicada, Universidade Federal do Rio de Janeiro, RJ. Retrieved August 20, 2007, from http:/ /teses.ufrj.br/ip_m/lucianamariarizodias.pdf

Ervin, R. A., Bankert, C. L., \& DuPaul, G. J. (1996). Treatment of attention-deficit/hiperactivity disorder. In M. A. Reinecke, F. M. Dattilio, \& A. Freeman (Eds.), Cognitive therapy with children and adolescents (pp. 38-61). New York: The Guilford Press.

Facion, J. R. (2004). Transtorno de déficit de atenção/ hiperatividade (TDAH): Atualização clínica. Revista de Psicologia da UnC, 1(2), 54-58. Retrieved August 20, 2006, from http://www.nead.uncnet.br/2004/revistas/psicologia/2/ 23.pdf

Freire, A. C. C., \& Pondé, M. P. (2005). Estudo piloto da prevalência de TDAH entre crianças escolares na cidade de Salvador, Bahia, Brasil. Arquivos de Neuropsiquiatria, 63(2), 474-478

Frison, L. M. B., \& Schwartz, S. (2002). Motivação e aprendizagem: Avanços na prática pedagógica. Ciências \& Letras Revista da Faculdade Porto Alegre, 32, 117-131.
Glass, C. S., \& Wegar, K. (2000). Teacher perceptions of the incidence and management of attention deficit hyperactivity disorder. Education, 121(2), 412-421.

Guardiola, A. (2006). Transtorno de atenção: Aspectos neurobiológicos. In N. T. Rotta, L. Ohlweiler, \& R. S. Riesgo (Eds.), Transtornos de aprendizagem: Abordagem neurobiológica e multidisciplinar (pp. 285-289). Porto Alegre, RS: Artes Médicas.

Havey, J. M. (2007). A comparison of Dutch and US teachers' perceptions of the incidence and management of ADHD. School Psychology International, 28(1), 46-52.

Havey, J. M., Olson, J. M., McCormick, C., \& Cates, G. L. (2005). Teachers' perceptions of the incidence and management of attention-deficit hyperactivity disorder. Applied Neuropsycholgy, 12(2), 120-127.

Landskron, L. M. F. (2007). As narrativas dos professores sobre $o$ TDAH. Dissertação de Mestrado não-publicada, Universidade Federal do Rio Grande do Sul, Porto Alegre, RS

Low, A. M. A. (2006). Diagnóstico neurofisiológico no tratamento da atenção. In N. T. Rotta, L. Ohlweiler, \& R. S. Riesgo (Eds.), Transtornos de aprendizagem: Abordagem neurobiológica e multidisciplinar (pp. 329-346). Porto Alegre, RS: Artes Médicas.

Marcon, M. S. (2006). Transtorno do déficit de atenção com hiperatividade (TDAH) e educação física. Revista Catarinense de Educação Física, 2. Retrieved May 15, 2007, from http://redebonja.cbj.g12.br/ielusc/revista_edf/ numero02.php

Poeta, L. S., \& Rosa Neto, F. (2004). Estudo epidemiológico dos sintomas do transtorno do déficit de atenção/ hiperatividade e transtornos de comportamento em escolares da rede pública de Florianópolis usando EDAH. Revista Brasileira de Psiquiatria, 26(3), 150-155.

Polanczyk, G., Lima, M. S., Horta, B. L., Biederman, J., \& Rohde, L. A. (2007). The worldwide prevalence of ADHD: A systematic review and metaregression análisis. American Journal of Psychiatry, 164(6), 942-948.

Riesgo, R. S. (2006). Transtorno da atenção: Comorbidades. In N. T. Rotta, L. Ohlweiler, \& R. S. Riesgo (Eds.), Transtornos de aprendizagem: Abordagem neurobiológica $e$ multidisciplinar (pp. 347-363). Porto Alegre, RS: Artes Médicas.

Rohde, L. A. (1999). Transtorno de déficit de atenção hiperatividade: $O$ que é? Como ajudar? Porto Alegre, RS: Artes Médicas,

Rohde, L. A. (2002). ADHD in Brazil: The DSM-IV criteria in a culturally different population. Journal of the American Academy of Child and Adolescent Psychiatry, 41, 1131-1133.

Rohde, L. A., Barbosa, G., Tramontina, S., \& Polanczyk, G. (2000). Transtorno do déficit de atenção e hiperatividade. Revista Brasileira de Psiquiatria, 22(2), 7-11.

Rohde, L. A., Biederman, J., Busnello, E. A., Zimmermann, H., Schmitz, M., Martins, S., et al. (1999). ADHD in a school sample of Brazilian adolescents: A study of prevalence, comorbid conditions, and impairments. Journal of the American Academy of Child and Adolescent Psychiatry, 38, 716-722.

Rohde, L. A, Dorneles, B. V., \& Costa, A. C. (2006). Intervenções escolares no transtorno de déficit de atenção/hiperatividade. In N. T. Rotta, L. Ohlweiler, \& R. S. Riesgo (Eds.), Transtornos de aprendizagem: Abordagem neurobiológica e multidisciplinar (pp. 365-374). Porto Alegre, RS: Artes Médicas. 
Rotta, N. T. (2006). Transtornos de atenção: Aspectos clínicos. In N. T. Rotta, L. Ohlweiler, \& R. S. Riesgo (Eds.), Transtornos de aprendizagem: Abordagem neurobiológica e multidisciplinar (pp. 301-313). Porto Alegre, RS: Artes Médicas.

Segenreich, D., \& Mattos, P. (2004). Eficácia da bupropiona no tratamento do TDAH. Uma revisão sistemática e análise crítica de evidências. Revista de Psiquiatria Clínica, 31(3), 117-123.

Silva, R. A., \& Paula Souza, L. A. (2005). Aspectos lingüísticos e sociais relacionados ao transtorno de déficit de atenção/ hiperatividade. Revista CEFAC, 7(3), 295-299.

Smith, C., \& Strick, L. (2001). Dificuldades de aprendizagem de A a Z. Porto Alegre, RS: Artes Médicas.

Spencer, T. J., Biederman, J., Wilens, T. E., \& Faraone, S.V. (2002). Overview and neurobiology of attention-deficit/ hyperactivity disorder. Journal Clinical Psychiatry, 63(12), 3-9.

Ulloa, C. S. (2007). La controversial Ritalina: ¿Como es su consumo en Costa Rica? Población y Salud en Mesoaméric, 4(2). Retrieved November 17, 2007, from http:// redalyc.uaemex.mx/redalyc/pdf/446/44640205.pdf

Vasconcelos, M., Werner, J., Jr., Malheiros, A. F. A., Lima, D .F. N., Santos, I. S. O, \& Barbosa, J. B. (2003). Prevalência do transtorno de déficit de atenção/hiperatividade numa escola pública primária. Arquivos de Neuropsiquiatria, 61, 67-73.

Vetter, V. L., Elia, J., Erickson, C., Berger, S., Blum, N., Uzark, K., et al. (2008). Cardiovascular monitoring of children and adolescents with heart disease receiving medications for attention deficit/hyperactivity disorder: A scientific statement from the American Hear Association Council on Cardiovascular Disease in the Young Congenital Cardiac Defects Committee and the Council on Cardiovascular Nursing. Circulation, 117, 2407-2423. Retrieved from http:/ / c i r c a h a jour n a $1 \mathrm{~s}$. or g/ c g i / r e p ri n t / CIRCULATIONAHA.107.189473 